\title{
REVISTA DE DIREITO ADMINISTRATIVO
}

ISSN 0034-8007 :: e-ISSN 2238-5177

\section{Pareceristas colaboradores do volume 279, número 1}

Alexandre Morais da Rosa - Universidade do Vale do Itajaí | Santa Catarina | RS | Brasil

Cristiane Silva Kaitel - Pontifícia Universidade Católica de Minas Gerais |Minas Gerais| MG | Brasil

Daniel Wunder Hachem - Universidade Federal do Paraná e Pontifícia Universidade Católica do Paraná | Paraná | PR | Brasil

Eduardo Reale Ferrari - Universidade de São Paulo | São Paulo | SP | Brasil

Elcio Nacur Rezende - Escola Superior Dom Helder Câmara e Faculdade Milton Campos | Minas Gerais | MG | Brasil

Emerson Gabardo - Universidade Federal do Paraná e Pontifícia Universidade Católica do Paraná | Paraná | PR | Brasil

Henrique Ribeiro Cardoso - Universidade Federal de Sergipe | Sergipe | SE | Brasil José Renato Gonçalves - Universidade de Lisboa | Lisboa | Portugal

Marcus Vinícius Aguiar Macedo - Universidade Federal do Rio Grande do Sul | Rio Grande do Sul | RS | Brasil

Miguel Assis Raimundo - Universidade de Lisboa | Lisboa | Portugal

Rita Calçada Pires - Universidade Nova de Lisboa | Lisboa | Portugal

Rui Manuel Pinto Soares Pereira Dias - Universidade de Coimbra | Coimbra | Portugal 